\title{
Synaptic Scaling and the Development of a Motor Network
}

\author{
Laura D. Knogler, ${ }^{1,2}$ Meijiang Liao, ${ }^{1}$ and Pierre Drapeau ${ }^{1}$ \\ ${ }^{1}$ Department of Pathology and Cell Biology and Le Groupe de Recherche sur le Système Nerveux Central, Université de Montréal, Montréal, Québec H3T \\ 1J4, Canada, and ${ }^{2}$ Graduate Program in Neurological Sciences, Department of Neurology and Neurosurgery, McGill University, Montréal, Québec H3A 2B4, \\ Canada
}

\begin{abstract}
Neurons respond homeostatically to chronic changes in network activity with compensatory changes such as a uniform alteration in the size of miniature postsynaptic current (mPSC) amplitudes termed synaptic scaling. However, little is known about the impact of synaptic scaling on the function of neural networks in vivo. We used the embryonic zebrafish to address the effect of synaptic scaling on the neural network underlying locomotion. Activity was decreased during development by TTX injection to block action potentials or CNQX injection to block glutamatergic transmission. Alternatively TNF $\alpha$ was chronically applied. Recordings from spinal neurons showed that glutamatergic mPSCs scaled up $\sim 25 \%$ after activity reduction and fortuitously scaled down $\sim 20 \%$ after TNF $\alpha$ treatment, and were unchanged following blockade of neuromuscular activity alone with $\alpha$-bungarotoxin. Regardless of the direction of scaling, immediately following reversal of treatment no chronic effect was distinguishable in motoneuron activity patterns or in swimming behavior. We also acutely induced a similar increase of glutamatergic mPSC amplitudes using cyclothiazide to reduce AMPA receptor desensitization or decrease of glutamatergic mPSC amplitudes using a low concentration of CNQX to partially block AMPA receptors. Though the strength of the motor output was altered, neither chronic nor acute treatments disrupted the patterning of synaptic activity or swimming. Our results show, for the first time, that scaling of glutamatergic synapses can be induced in vivo in the zebrafish and that synaptic patterning is less plastic than synaptic strength during development.
\end{abstract}

\section{Introduction}

A key feature of synapses is their ability to undergo presynaptic and/or postsynaptic modifications during development and in response to experience and environmental changes. While Hebbian plasticity is known to act rapidly at individual synapses to alter the efficiency of specific neuronal connections (reviewed by Malenka and Bear, 2004), other plasticity mechanisms must integrate these changes over a longer time scale so that synapses onto a given cell are changed accordingly to allow for the net input to remain constant (Miller, 1996). Several forms of homeostatic synaptic plasticity have been described, the best-characterized example of which is synaptic scaling where the strength of all synapses onto a neuron are multiplicatively scaled in a direction that opposes chronic changes in activity (Turrigiano et al., 1998). Most investigations into synaptic scaling have been done in vitro or by computational modeling, leaving unanswered the significance of these changes in biologically relevant processes such as development, learning, or disease (Horn et al., 1996; Rabinowitch and Segev, 2008; Turrigiano, 2008; Savioz et al., 2009). Furthermore, of the investigations of glutamatergic synaptic scaling in vivo (Desai et al., 2002; Pawlak et al., 2005; Gonzalez-Islas and Wenner, 2006; Echegoyen et

Received Feb. 17, 2010; revised May 18, 2010; accepted May 24, 2010.

This work was funded by a grant (P.D.) from the Canadian Institutes of Health Research and the Groupe de Recherche sur le Système Nerveux Central, and a studentship (L.D.K.) from the Natural Sciences and Engineering Research Council of Canada. We thankH. Okamoto for the /s/7-GFP transgenic line, L. Saint-Amant for suggesting the bungarotoxin experiment, and L. Saint-Amant, G. Armstrong, and E. Brustein for critiques of the manuscript.

Correspondence should be addressed to Dr. Pierre Drapeau, Department of Pathology and Cell Biology, Université de Montréal, C.P. 6128, Succ. Centre-ville, Montréal, Québec H3C 3J7, Canada. E-mail: p.drapeau@umontreal.ca. DOI:10.1523/JNEUROSCI.0880-10.2010

Copyright $\odot 2010$ the authors $\quad 0270-6474 / 10 / 308871-11 \$ 15.00 / 0$ al., 2007; Kaneko et al., 2008), there are very few (Mongeon et al., 2008; Whiting et al., 2009) that also looked at synaptic scaling at glycinergic synapses despite their known role in learning and memory processes (Gaiarsa et al., 2002) and proper patterning of the motor central pattern generator of the spinal cord (reviewed by Roberts et al., 2008).

To better understand the characteristics of synaptic scaling and how this affects functional network activity and behavior in vivo, we focused on measuring changes at synaptic, network and behavioral levels using the developing zebrafish (Danio rerio) motor network as an experimentally accessible model. Development of the zebrafish motor network is rapid, as at $18 \mathrm{~h}$ postfertilization (hpf) the embryo shows spontaneous coiling, at $27 \mathrm{hpf}$ a robust touch response can induce swimming events, and by 52 hpf the embryo has hatched and exhibits swimming behavior (Saint-Amant and Drapeau, 1998; Drapeau et al., 2002). If synaptic scaling is truly a homeostatic process during development, then the changes in synaptic strength following activity perturbation should serve to stabilize this behaviorally relevant network activity.

We tested this hypothesis in vivo by examining miniature spontaneous synaptic activity, integrated motoneuron inputs, their output to muscle fibers, and the consequent swimming behavior following induction of synaptic scaling. Despite a significant scaling up or down of glutamatergic (but not glycinergic) synapses in spinal neurons following chronic activity blockade or TNF $\alpha$ exposure, respectively, the strength of the motor output was altered but network activity patterns and overall motor behavior did not differ from controls. Furthermore, acute pharmacological manipulations to increase or decrease synaptic amplitudes and the 
strength of the motor output to a similar extent as observed during chronic synaptic scaling failed to disrupt the pattern of network activity. We conclude that the spinal network can manifest synaptic scaling but the network nonetheless develops a robust activity pattern.

\section{Materials and Methods}

Zebrafish maintenance. Zebrafish (Danio rerio) were bred and maintained at $28.5^{\circ} \mathrm{C}$ according to standard procedures (Westerfield, 1995). All experiments were performed in compliance with the guidelines of the Canadian Council for Animal Care and conducted at the University of Montreal.

Pharmacological injections. Wild-type or transgenic embryos (Isl1::GFP transgenic line, gift from H. Okamoto, RIKEN Brain Science Institute, Wako, Japan) were anesthetized in 0.04\% tricaine (MS-222; Sigma) dissolved in modified Evans (see later), dechorionated, and embedded in a $1 \%$ solution of low-melting-point agarose. Embryos were injected at 17, 24, 48, or $72 \mathrm{hpf}$ with either vehicle or the following concentrated doses of drugs to allow for subsequent dilution in the embryo: $250 \mu \mathrm{M}$ 6-cyano-7nitroquinoxaline-2,3-dione (CNQX; Sigma), $250 \mu \mathrm{m}$ tetrodotoxin (TTX; Tocris Bioscience), $600 \mu \mathrm{M}(2 R)$-amino-5-phosphonovaleric acid (AP-5; Sigma), or $200 \mu \mathrm{M} \alpha$-bungarotoxin ( $\alpha$-BgTx; Sigma). Solutions were injected as a bolus into the brain (up to $30 \mathrm{hpf}$ ) or heart (after $30 \mathrm{hpf}$ ) using a fine glass electrode connected to a Picospritzer III (General Valve). Injection solutions contained 1\% Fast Green for visual control. Embryos were then removed from the agarose and raised as usual. To verify the efficacy of the injections, touch response was assessed over time following injections. Control-injected fish recovered within minutes, whereas fish injected with drugs did not recover for several hours if at all. Thus fish responding to touch within minutes following pharmacological injection were excluded $(<5 \%)$, as well as any fish displaying unusual morphology $(<5 \%)$.

Neural recordings. Zebrafish were anesthetized in $0.04 \%$ tricaine dissolved in modified Evans solution containing the following (in $\mathrm{mM}$ ): 134 $\mathrm{NaCl}, 2.9 \mathrm{KCl}, 2.1 \mathrm{CaCl}_{2}, 1.2 \mathrm{MgCl}_{2}, 10 \mathrm{HEPES}$, and 10 glucose, adjusted to $290 \mathrm{mOsm}$ and $\mathrm{pH} 7.8$, and dissected according to previously described procedures (Drapeau et al., 1999). Briefly, spinal neurons in somites $10-15$ were selected for recording based on soma size and position as visualized by oblique illumination (Olympus BX61W1). All experiments and manipulations were performed at room temperature.

To record mPSCs, $15 \mu \mathrm{M}$ D-tubocurarine (Sigma) was added to the Evans solution to fully block neuromuscular transmission. Electrophysiological recordings were done in the presence of $0.5 \mu \mathrm{M}$ TTX and $1 \mu \mathrm{M}$ strychnine to isolate glutamatergic mPSCs, or in $0.5 \mu \mathrm{M}$ TTX, $10 \mu \mathrm{M}$ CNQX, and $50 \mu \mathrm{M} \mathrm{AP-5}$ to isolate glycinergic mPSCs. Patch-clamp electrodes for spinal neurons (5-12 M $\Omega$ ) pulled from thin-walled Kimax-51 borosilicate glass and were filled with the following intracellular solution (in mM): 105 D-gluconic acid, $16 \mathrm{KCl}, 2 \mathrm{MgCl}_{2}, 10 \mathrm{HEPES}$, and 10 EGTA, adjusted to pH 7.2, 290 mOsm. Sulforhodamine B (0.1\%; Sigma) was also included in the patch solution to label the cells and confirm their identity after a recording. Standard whole-cell recordings from 3-4 dpf larvae were obtained using an Axopatch 200B and a Molecular Devices CV 203BU headstage amplifier (Molecular Devices). Data were acquired at $40 \mathrm{kHz}$ and low-pass filtered at $5 \mathrm{kHz}$. Cells were held near their resting potential at $-65 \mathrm{mV}$ under voltage clamp unless otherwise specified. A maximum of three neural recordings were obtained from each larva.

Intrinsic excitability. Measurements of intrinsic cellular excitability were made with whole-cell current-clamp recordings performed in a TTX-free recording solution. Current was injected into the cell as a continuously increasing ramp $(0-500 \mathrm{pA}, 100 \mathrm{~ms}$ duration) to measure the minimum required (rheobasic) current to produce an action potential. Spike threshold was also calculated with these data by finding the difference between the voltage threshold for firing and the resting membrane voltage. A plot of action potential firing frequency versus current was made by injecting current into the cell in steps ( $25 \mathrm{pA}$ steps up to $375 \mathrm{pA}, 100 \mathrm{~ms}$ duration).

NMDA-induced slow oscillations. Measurements of the frequency of neuronal slow oscillations (related to episodes of swimming) were made by perfusing $200 \mu \mathrm{M}$ NMDA into the extracellular solution, which causes gradual membrane depolarizations of up to $20 \mathrm{mV}$ and induces persis- tent rhythmic activity (McDearmid and Drapeau, 2006). To compensate for the depolarization, up to $60 \mathrm{pA}$ of negative current was injected to maintain the troughs of oscillation at $-65 \mathrm{mV}$ and amplify this part of the activity. Data were analyzed by quantifying the number of complete episodes in a $40 \mathrm{~s}$ period, yielding a measure of the slow oscillation frequency.

Muscle recordings. Standard whole-cell recordings using 2-5 M $\Omega$ electrodes were obtained from slow-twitch (embryonic red) muscle fibers identified visually by their proximity to the surface and longitudinal orientation (Buss and Drapeau, 2000). In these recordings the concentration of Dtubocurarine was reduced to $\sim 2-3 \mu \mathrm{M}$ to prevent muscle contractions while only partially blocking neuromuscular transmission. The ryanodine receptor antagonist $20 \mu \mathrm{M} \mathrm{N}$-benzyl-p-toluenesulfonamide (Sigma) in $0.1 \%$ DMSO was also added to muscle recordings at $4 \mathrm{dpf}$ to prevent muscle contractions. This treatment did not appear to alter neural activity. Fictive swimming patterns in the muscle were either spontaneous or evoked by turning a light on and off. Recordings were analyzed for episode duration, burst duration, burst frequency, synaptic peaks related to the number of contractions per burst, their frequency, maximum amplitude, and percentage time active.

Acute pharmacological treatment. CNQX (1.2-2.4 $\mu \mathrm{M})$ or cyclothiazide (CTZ; 7-10 $\mu \mathrm{M}$; Sigma) was dissolved in Evans and perfused into the recording bath after collecting $5 \mathrm{~min}$ of baseline whole-cell neuron or muscle recordings. Data representing the acute CNQX and CTZ treatments were collected from 5 min following the start of the drug application onward.

Analysis. Electrophysiological analyses were performed offline using Clampex 10.2 and Clampfit 10.2 software (Molecular Devices). mPSC recordings (lasting $10 \mathrm{~min}$ ) were analyzed using a template to collect the first consecutive 50-200 mPSCs starting 100 s into the recording to allow the patch to stabilize. All mPSCs were verified visually before inclusion and mPSCs from each cell were averaged for comparison of amplitudes and frequencies. Measurements of intrinsic excitability, slow oscillation frequency, and muscle fiber parameters were measured by hand using program cursors.

SigmaStat 3.5 (Systat Software) was used to assess data for statistical significance. Statistical analyses used one-way or two-way ANOVA grouped by day and experimental treatment to ensure that there was no interaction effect and thus data could be pooled across days. For paired datasets, the paired Student's $t$ test was used. For nonparametric statistical testing, we used the Mann-Whitney rank sum test. Significance was assessed at $p<0.05$, as indicated by one asterisk. Two asterisks indicate $p<0.01$, and three asterisks indicate $p<0.001$. All data are represented as mean \pm SEM. All individual mPSCs from each condition were also plotted on a cumulative histograms using Kaleidagraph 3.1 software.

Tnf $\alpha$ expression. RT-PCR from cDNA pools reverse transcribed from RNA isolated from $18,24,48,72,96$, and 120 hpf wild-type zebrafish was performed to examine the endogenous expression of $\operatorname{tnf} \alpha$ (GenBank accession: AY427649) during development using the following primer pair: $\left(5^{\prime}\right)$ ACGTCTGAACTGACTGAGGAACA and (3') GCCAAGAGTGTATGATAGAGGTCA (Invitrogen). $\operatorname{tnfb}$ (as a control for cDNA quality) was also amplified using the following primer pair: (5') GTACCTGAGCCACACCATCAATC and (3') AGTGCCCTTGTTATAGTGCTCTTG. Fragments were cloned into pGEM-T Easy cloning vector (Promega) and sequenced to confirm identity.

Recombinant zebrafish TNF $\alpha$ was produced from $t n f \alpha$ mRNA synthesized in vitro. Briefly, mRNA from zebrafish embryos was isolated and reverse transcribed to make cDNA that was subcloned into the pCS2 vector at the BamHI and XhoI sites and the mRNA was synthesized by the mMESSAGE mMACHINE high-yield capped RNA transcription kit (Ambion). tnf $\alpha$ cDNA was inserted into a pQE30 vector by BamHI and PstI fusion with 6his tag. Purification of TNF $\alpha$ protein under native conditions was done by protocols 9 and 12 of QIAexpressionist (Qiagen). Dose-response experiments showed high survival rates and lower concentrations proved just as effective as higher concentrations in preliminary experiments; therefore, we chose to inject $1 \mu \mathrm{M} \mathrm{TNF} \alpha$ at $1-2 \mathrm{dpf}$ as described above for a final concentration of $\sim 50 \mathrm{~nm}$ after dilution in the embryos, a concentration shown to acutely induce synaptic scaling in vitro (Stellwagen et al., 2005). 
Confocal microscopy. To investigate changes in number of neurons across treatments, fixed Isl1-GFP transgenic embryos were imaged using a Quorum WaveFX spinning disk system (Quorum Technologies Inc) based on a modified Yokogawa CSU-10 head (Yokogawa Electric) mounted on an upright Olympus BX61W1 fluorescence microscope and connected to a Hamamatsu ORCA-ER camera. Briefly, a set of stacked $Z$-series images were collected using Volocity software (Improvision) and the number of $\mathrm{GFP}^{+}$neurons per somite was counted per embryo using ImageJ.

Behavioral recordings. Responses to touch at $24 \mathrm{hpf}$ were evoked by tapping the fish lightly on the tail with forceps and quantified as either responding or not. These measurements were repeated at regular intervals to determine the time course of recovery of touch response following injection. Swimming was measured at either 3 or $4 \mathrm{dpf}$ to parallel different experimental paradigms that elicited synaptic scaling. Swimming behavior in larval zebrafish transitions rapidly from sustained burst swimming at 2-3 dpf to a more mature beat-and-glide pattern by $4 \mathrm{dpf}$ (Buss and Drapeau, 2001). Therefore it was most informative to measure tail contraction frequency and free-swimming velocity at $3 \mathrm{dpf}$, when burst swimming is still observed, and to measure tail contraction frequency and beat duration at $4 \mathrm{dpf}$, when discrete "beat" periods are present (Buss and Drapeau, 2001).

To measure tail contraction frequency (Buss and Drapeau, 2001), larvae were embedded in 1\% low-melting-point agarose (Invitrogen) dorsal side up with the tail free to move. Episodes of tail alternations representative of swimming were elicited by a light tap on the tail with forceps. Movements were filmed at $60 \mathrm{~Hz}$ with a Point Gray Research (PGR) Grasshopper 2 camera mounted on a Zeiss dissection microscope. A minimum of three episodes of swimming were obtained from each fish to be averaged. Frequency of tail contractions was measured offline by counting the number of full tail contractions per distinct bout of swimming, thus accounting for differences in contraction frequency at the beginning versus end of a swimming bout. Duration of individual swimming beat periods at $4 \mathrm{dpf}$ was also measured and averaged across fish in each condition before statistical analysis.

To measure swimming velocity, free-swimming $3 \mathrm{dpf}$ larvae were individually placed in a large dish filled with egg water at room temperature and filmed at $15 \mathrm{~Hz}$ with a PGR Flea 2 camera. The larva was touched lightly on the tail to initiate swimming. Swimming velocity was calculated from the change in position of the larva's head in each frame divided by the duration of swimming using the "manual tracking" plug-in for ImageJ software. Velocity calculations did not include periods of swimming in which the larva was touching or alongside of the wall of the dish.

All video recordings were made using PGR FlyCap software and all video analyses were done using ImageJ software. Data were averaged across all embryos in each experimental group to obtain means for comparison between conditions and statistically analyzed as described for neural and muscle recordings.

\section{Results}

As synaptic scaling has not been previously studied in zebrafish, we first sought to determine whether and under what conditions it could be induced in living embryos and posthatching larvae. Electrophysiological and behavioral experiments were performed mostly on 3 or $4 \mathrm{dpf}$ zebrafish larvae that were injected with drugs as early as $17 \mathrm{hpf}$, allowing a few days for chronic changes to occur. We started by testing the effects of TTX or CNQX treatment, which reduced activity and caused upward scaling, and later those of TNF $\alpha$ treatment, which caused downward scaling.

\section{Scaling up occurs at glutamatergic synapses upon activity blockade in the zebrafish embryo}

The first report of synaptic scaling used chronic TTX treatment of cultured cortical neurons to block action potentials globally, and this paradigm has since proved to be a robust way to elicit synaptic scaling in other systems (Turrigiano et al., 1998). We therefore sought to test the effects of TTX on synaptic activity in zebrafish motoneurons. To investigate the in vivo effects of drug treatment on developing zebrafish, drugs had to be injected directly into the embryo, as described in the Materials and Methods. To confirm the efficacy of this method, touch response (Saint-Amant and Drapeau, 1998) was measured after injection. Injections of TTX $250 \mu \mathrm{M}$, diluted $\sim 20$-fold in the $24 \mathrm{hpf}$ embryo, resulted in complete paralysis that never recovered up to $6 \mathrm{dpf}$, thus confirming an effective block of motor activity as previously reported for neural activity (Saint-Amant and Drapeau, 2001). Additionally, while control embryos recovered from their sham injection within 10 min to show a touch response, only 50\% of CNQXinjected ( 250 or $500 \mu \mathrm{M}$ ) embryos showed a touch response after $2 \mathrm{~h}$, and $100 \%$ only after $5-6 \mathrm{~h}$ postinjection. Therefore CNQX significantly reduced activity in comparison to control, though not as persistently as for TTX, possibly indicating a developmental window for its effect. None of these injections resulted in gross morphological abnormalities.

Zebrafish embryos and larvae show two types of swim-related spinal cord synaptic activity: glutamatergic and glycinergic (Buss and Drapeau, 2001; Saint-Amant and Drapeau, 2001), and we therefore examined each type. Electrophysiological recordings of isolated glutamatergic mPSCs (in the presence of acutely applied TTX and strychnine) were obtained from spinal neurons $(n=$ 132) in $4 \mathrm{dpf}$ larvae following various time intervals of chronic TTX treatment. Interestingly, we were able to consistently induce synaptic scaling only after a minimum of $48 \mathrm{~h}$ of TTX treatment when embryos were treated at $2 \mathrm{dpf}$ (control, $n=13$; TTX, $n=7$; amplitudes for control and TTX were $17.5 \pm 3$ and $20.9 \pm 0.9 \mathrm{pA}$, respectively; $p<0.05)$, or a minimum of $72 \mathrm{~h}$ when embryos were treated at $1 \mathrm{dpf}$ (control, $n=17$; TTX, $n=17$; amplitudes for control and TTX were $18.0 \pm 1$ and $24.2 \pm 2 \mathrm{pA}$, respectively; $p<$ 0.05 ) (summarized in Fig. 1). Synaptic scaling induced by these two treatments were comparable; thus, data from these conditions were grouped. No synaptic scaling was seen following $48 \mathrm{~h}$ of TTX treatment between 1 and $3 \mathrm{dpf}$ (control, $n=20$; TTX, $n=$ 23; amplitudes for control and TTX were $22.4 \pm 1$ and $21.0 \pm 0.7$ $\mathrm{pA}$, respectively; $p=0.31$ ), or after only $24 \mathrm{~h}$ of TTX treatment at any age tested (control, $n=23$; TTX, $n=22 ; p>0.80$ for all time intervals) (Fig. 1).

Recordings following TTX treatment from 1 or $2 \mathrm{dpf}$ to $4 \mathrm{dpf}$ showed that synapses were significantly scaled up by an average $\sim 30 \%$ in comparison to amplitudes from vehicle-injected controls (Fig. 2A) (control, $n=24$; TTX, $n=30$; amplitudes for control and TTX were $18.0 \pm 1.4$ and $23.4 \pm 1.3 \mathrm{pA}$, respectively; $p<0.01)$. This change in amplitude was not accompanied by any changes in glutamatergic mPSC frequency $(p=0.33)$ or time course (Fig. 2A). Furthermore, a cumulative histogram showed that when a scaling factor was applied, the TTX distribution was almost perfectly superimposable on the control distribution (Fig. $2 \mathrm{~A}$ ), indicating that every synapse was scaled multiplicatively in proportion to its original strength, a hallmark of synaptic scaling.

Furthermore, significantly increased glutamatergic mPSC amplitudes were also seen following CNQX treatment using the same timing of injections ( 1 or 2 to $4 \mathrm{dpf}$ ) as was used to induce synaptic scaling with TTX treatment (Fig. $2 B$ ). These amplitudes were on average $\sim 20 \%$ larger than vehicle-injected controls (control, $n=17$; CNQX, $n=22$; amplitudes for control and CNQX were $21.0 \pm 1.2$ and $25.6 \pm 1.3 \mathrm{pA}$, respectively; $p<0.05$ ), and also showed evidence of multiplicative scaling (Fig. 2 B). Glutamatergic mPSC frequency after CNQX treatment was unchanged from control values $(p=0.90)$ as was the time course (Fig. 2B). Pooled data from TTX and CNQX experiments 
showed that the degree of scaling induced was comparable between identified MNs and INs (MNs, $n=18$; INs, $n=15 ; p=$ $0.50)$. In contrast to the effects of TTX and CNQX, embryos injected with AP-5 showed no significant differences from vehicle-injected controls in glutamatergic mPSC amplitude (control, $n=9$; AP-5, $n=13 ; p=0.37$ ) (Fig. $2 C$ ), time course, or frequency $(p=0.82)$. This was in accord with previous literature that showed no effect of chronic AP-5 exposure on glutamatergic $\mathrm{mPSC}$ amplitudes (Turrigiano et al., 1998). These experiments thus support the role of bona fide synaptic scaling in vivo in the embryonic zebrafish spinal cord in response to activity perturbation, either completely with TTX, or transiently with CNQX.

To determine whether the increase in glutamatergic mPSC amplitudes following TTX or CNQX treatment was due to a reduction in electrical activity within the CNS rather than as a generalized consequence of altered muscle activity, embryos were injected at $1 \mathrm{dpf}$ with a high dose of $\alpha$-BgTx to selectively block muscle nicotinic acetylcholine receptors, resulting in total muscle paralysis but an otherwise normal CNS level of activity. Whole-cell patchclamp recordings of glutamatergic mPSC amplitudes at $4 \mathrm{dpf}$ from $\alpha$-BgTx-treated larvae were not significantly different from vehicle-injected controls (control, $n=5$; $\alpha$-BgTx, $n=4$; control and $\alpha$-BgTx amplitudes were $21.2 \pm 2.2$ and $20.5 \pm 0.9 \mathrm{pA}$, respectively; $p=0.77$ ), showing that the synaptic scaling seen with TTX and CNQX treatment was dependent on electrical activity within the CNS and not the absence of movement.

\section{Absence of synaptic scaling at glycinergic synapses}

As discussed previously, despite the prevalence of glycinergic synapses in the spinal cord, synaptic scaling has been predominantly investigated at glutamatergic synapses. Interestingly, the same timing of TTX and CNQX treatments ( 1 or 2 to 4 $\mathrm{dpf}$ ) that elicited synaptic scaling in glutamatergic mPSCs described above was unable to induce significant scaling of glycinergic mPSC amplitudes (Fig. $3 A, B$ ) (TTX treatment, $p=0.24$; CNQX treatment, $p=0.60)$. While there was a trend toward smaller glycinergic mPSC amplitudes, which was particularly significant in the presence of CNQX, the amplitudes varied considerably and cumulative histograms showed an uneven decrease that was not indicative of multiplicative synaptic scaling and therefore not similar to

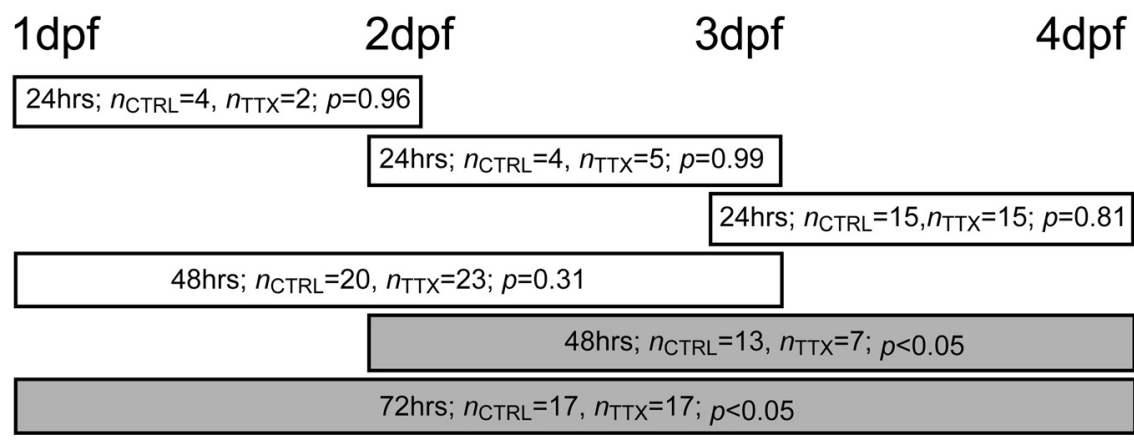

Figure 1. A significant increase in glutamatergic mPSC amplitude was only seen following chronic blockade of network activity with TTX from 1 or $2 \mathrm{dpf}$ to $4 \mathrm{dpf}$. Boxed text refers to TTX treatment duration starting at time of injection and ending at time of recording, number of recordings in control and treated larvae, and significance of mPSC amplitude change.
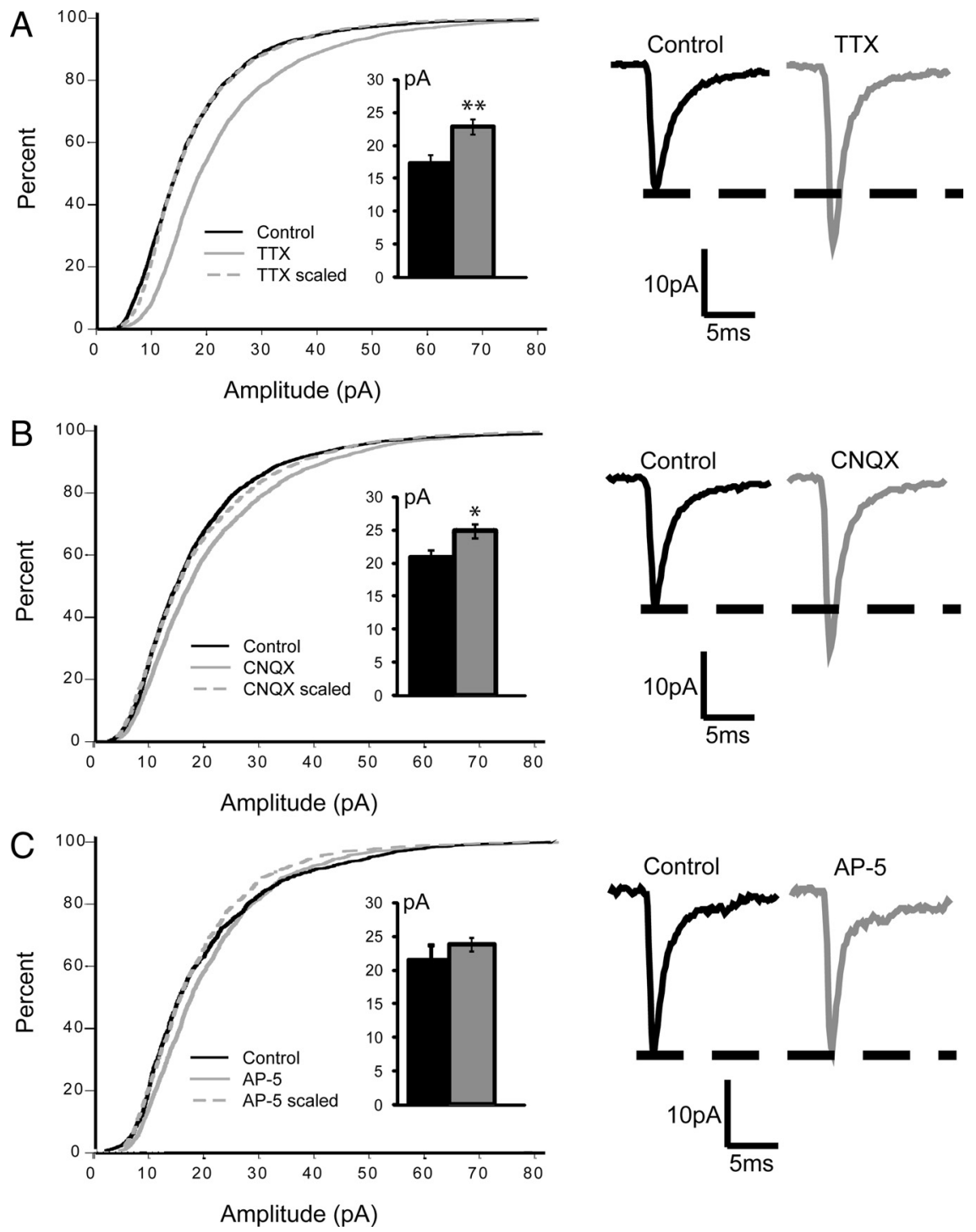

Figure 2. Chronic TTX or CNQX treatment results in a scaling up of glutamatergic mEPSC amplitudes at $4 \mathrm{dpf}$. A, Left, Cumulative histograms of glutamatergic mEPSC amplitudes from control $(n=24)$ and TTX-treated $(n=30)$ embryos ( $\geq 50$ events per neuron). Inset, Average mEPS (amplitude for the same conditions. Right, Average mEPS ( waveforms of one neuron for the same conditions. B, Left, Cumulative histograms of glutamatergic mEPSC amplitudes from control ( $n=17)$ and CNQX-treated $(n=22)$ embryos. Inset, Average mEPSC amplitude for the same conditions. Right, Average mEPSC waveforms of one neuron for the same conditions. C, Left, Cumulative histograms of glutamatergic mEPSC amplitudes from control $(n=9)$ and AP-5-treated $(n=13)$ embryos. Inset, Average mEPSC amplitude for the same conditions. Right, Average mEPSC waveforms of one neuron for the same conditions. All data here and below are reported as mean \pm SEM for the number of neurons indicated; two-way ANOVA, ${ }^{*} p<0.05,{ }^{* *} p<0.01$. 

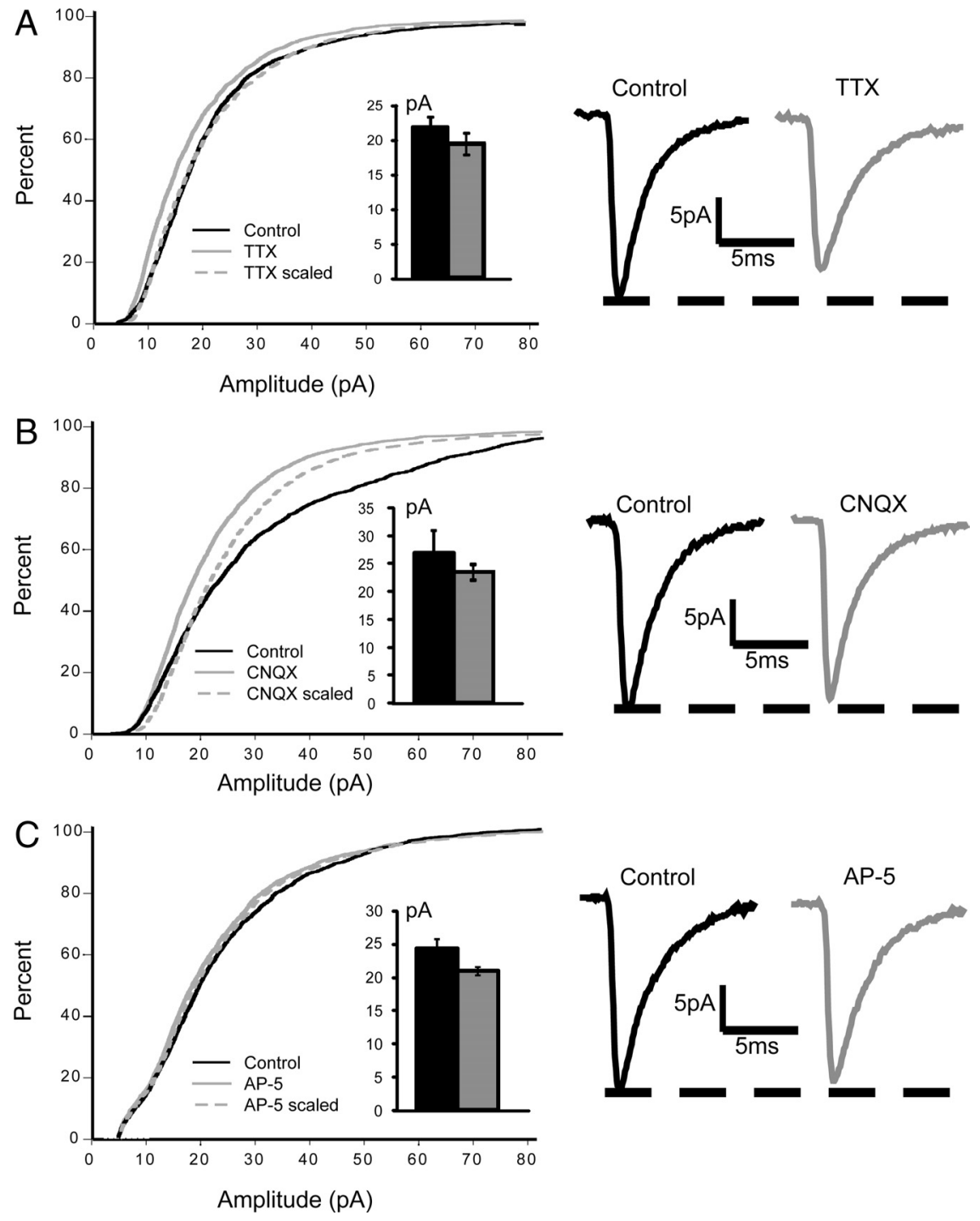

Figure 3. Chronic TTX, CNQX, or AP-5 treatment does not scale glycinergic mIPSC amplitudes at $4 \mathrm{dpf}$. $A$, Left, Cumulative histograms of glycinergic mIPSC amplitudes from control $(n=11)$ and TTX-treated $(n=12)$ embryos. Inset, Average mIPSC amplitude for the same conditions. Right, Average mIPSC waveforms of one neuron for the same conditions. $\boldsymbol{B}$, Left, Cumulative histograms of glycinergic mIPSC amplitudes from control $(n=11)$ and CNQX-treated $(n=16)$ embryos. Inset, Average mIPSC amplitude for the same conditions. Right, Average mIPSC waveforms of one neuron for the same conditions. $C$, Left, Cumulative histograms of glycinergic mIPSC amplitudes from control $(n=9)$ and AP-5-treated $(n=10)$ embryos. Inset, Average mIPSC amplitude for the same conditions. Right, Average mIPSC waveforms of one neuron for the same conditions.

TNF $\alpha$ induces synaptic scaling down

As TNF $\alpha$ plays a significant and biologically relevant role in promoting synaptic scaling (Stellwagen et al., 2005; Stellwagen and Malenka, 2006; Kaneko et al., 2008), we sought to test its effects in the zebrafish embryo. Before testing the effect of manipulating TNF $\alpha$ levels, we first investigated the expression of endogenous TNF $\alpha$ in the embryonic zebrafish with RT-PCR at 18, 24, 48, 72,96 , and $120 \mathrm{hpf}$. Bands corresponding to the amplified sequence were clearly seen at all time points tested, with the exception of low or absent expression at $22 \mathrm{hpf}$ (Fig. 4A) $(n=3)$, and these bands were confirmed to be TNF $\alpha$ upon sequencing.

We produced recombinant zebrafish TNF $\alpha$ protein (see Materials and Methods) and injected it into $24 \mathrm{hpf}$ embryos at high concentrations (1-5 $\mu \mathrm{M})$, and the injected embryos showed an excellent survival rate $(>90 \%)$. We timed our injections of TNF $\alpha$ to follow the same parameters as used to induce synaptic scaling with TTX and CNQX treatment (2 dpf to $4 \mathrm{dpf}$ ), but electrophysiological recordings of glutamatergic mPSCs failed to show any significant changes in amplitude that would be indicative of synaptic scaling (control, $n=6$; TNF $\alpha, n=7$; amplitudes for control and TNF $\alpha$ were $19.6 \pm 2$ and $19.0 \pm 2 \mathrm{pA}$, respectively; $p=0.79$ ). However, varying the time points of injection and recording revealed that glutamatergic mPSCs were significantly and surprisingly scaled down by $\sim 20 \%$ following treatment with $\mathrm{TNF} \alpha$ protein from 1 or $2 \mathrm{dpf}$ to $3 \mathrm{dpf}$ (Fig. $4 B, C$ ) (control, $n=27$; TNF $\alpha, n=32$; amplitudes for control and TNF $\alpha$ were $22.6 \pm 0.9$ and $18.0 \pm 0.5 \mathrm{pA}$, respectively; $p<0.001)$. A cumulative histogram showed that this scaling was indeed a multiplicative phenomenon (Fig. 4B). As before, the time course of the glutamatergic mPSCs did not change (Fig. 4C).

However, the frequency of glutamatergic mPSCs was significantly reduced by

the effects seen with glutamatergic mPSCs (Fig. $3 A, B$ ). Furthermore, these treatments with TTX or CNQX resulted in no significant differences in glycinergic mPSC time course (Fig. $3 A, B$ ) or frequency (TTX treatment, $p=0.82$; CNQX treatment, $p=$ 0.36). Following AP-5 treatment, there was significant variation in mPSC amplitudes and frequencies between different experimental days (Fig. $3 C$ ) (control, $n=9$; AP-5, $n=10$; interaction effect between day and condition $p<0.001$ for amplitude and frequency).

To summarize, our experimental manipulations with TTX and CNQX caused a significant, multiplicative increase in glutamatergic but not in glycinergic mPSC amplitudes or frequency. Our results suggest that the properties of glycinergic and glutamatergic mPSCs are regulated via different mechanisms under these conditions. $\sim 20 \%$ following TNF $\alpha$ treatment (Fig. $4 D$ ) (frequencies for control and TNF $\alpha$ were $0.11 \pm 0.01$ and $0.09 \pm 0.01 \mathrm{~Hz}$, respectively; $p<0.05$ ), perhaps a result of smaller events being lost in the baseline noise. A reduction in glutamatergic mPSC frequency could also be representative of a loss of synapses, or even of neurons. Isl1::GFP transgenic zebrafish injected with TNF $\alpha$ at $2 \mathrm{dpf}$ were imaged by confocal microscopy at $3 \mathrm{dpf}$ to investigate possible changes in the number of spinal neurons. Cell counts showed no significant difference in GFP-positive cells per somite, with an average of $42-45$ per somite (Fig. $4 D$ ) (control, $n=7$; TNF $\alpha, n=6$; $p=0.69$ ), suggesting that neuronal numbers remained the same but leaving the possibility that synapse number had decreased, though we have no measure of synaptic numbers that would be of sufficient sensitivity to detect such a small (20\%) change. 
Using the same TNF $\alpha$ treatment protocol that elicited synaptic scaling in glutamatergic mPSCs (injection at 1-2 dpf, recording at $3 \mathrm{dpf}$ ), we investigated glycinergic mPSC characteristics, but we found no significant differences between the amplitude of glycinergic mPSCs from TNF $\alpha$-treated embryos and controls (control, $n=5$; TNF $\alpha, n=5 ; p=0.79$ ). A cumulative histogram showed no clear shift of the amplitude distribution in either direction; neither were there any changes in mPSC time course (Fig. $4 E$ ). Additionally, the frequency of glycinergic mPSCs under this experimental condition was comparable to control $(p=0.79)$.

To summarize, while we could robustly elicit synaptic scaling in glutamatergic mPSCs with TTX, CNQX, or TNF $\alpha$ treatment, no changes were seen in glycinergic mPSC amplitude or time course or in the frequency of either type of mPSC following any of these treatments.

\section{No changes in intrinsic} cellular excitability

Although the spinal network underlying locomotion in the zebrafish embryo appears to be synaptically driven (Buss and Drapeau, 2000) rather than due to intrinsic rhythmic neural firing properties (Buss et al., 2003), studies in the chick embryo have shown that intrinsic changes in cellular excitability can accompany, or precede, changes in synaptic strength (Wilhelm et al., 2009). We therefore examined intrinsic excitability in $3 \mathrm{dpf}$ larvae treated with TNF $\alpha$ and in $4 \mathrm{dpf}$ larvae treated with CNQX, the different stages at which scaling down and scaling up, respectively, of mPSCs were observed.

Electrophysiological recordings of spinal neurons under current clamp from TNF $\alpha$-treated larvae showed that no significant changes in intrinsic cellular excitability from control larvae at $3 \mathrm{dpf}$ as measured by average rheobase current (Fig. $5 A$ ) $(p=0.08)$, spike threshold (Fig. $5 B)(p=0.79)$, and frequency versus current $(f-I)$ plot (Fig. $4 D$ ) (control, $n=11$; TNF $\alpha, n=$ $10 ; p>0.05$ for all frequency data pairs at a given current step). Likewise, no significant differences were found at $4 \mathrm{dpf}$ between CNQX-treated and control larvae for average rheobase current (Fig. 5A) $(p=0.32)$, spike threshold (Fig. $5 B)(p=0.77)$, or frequency versus current $(f-I)$ plot (Fig. $5 C, D)$ (control, $n=6$; CNQX, $n=8 ; p>0.05$ for all frequency data pairs at a given current step). These results suggest that synaptic rather than intrinsic modes of homeostatic plasticity dominated under these experimental conditions.

\section{Homeostatic plasticity of network activity and motor behavior}

Beyond synaptic scaling and intrinsic excitability, homeostatic plasticity may be measurable as changes in network activity that arise from the integration of cellular and synaptic properties.
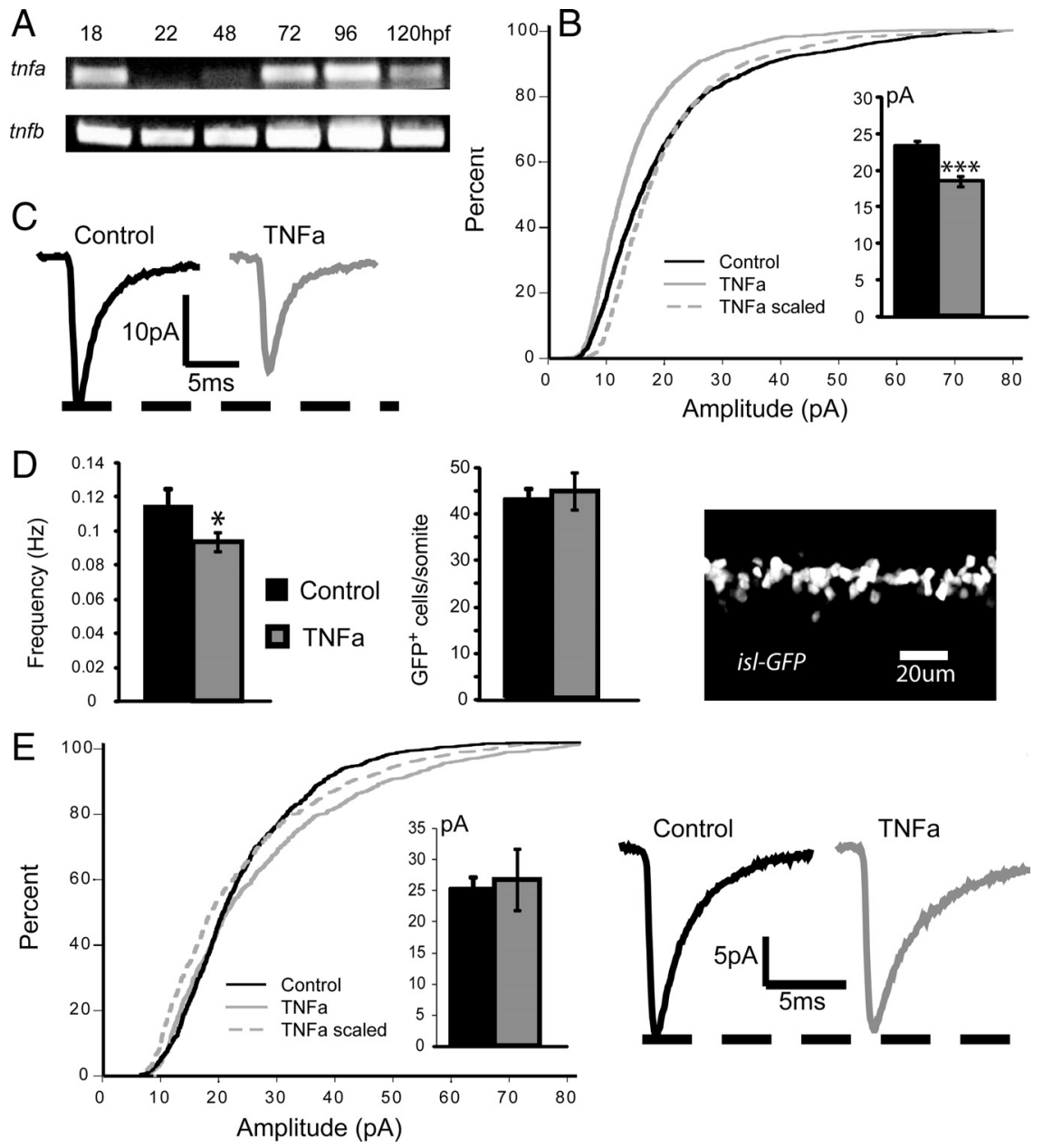

Figure 4. Chronic TNF $\alpha$ treatment results in a scaling down of glutamatergic mEPSC amplitudes at 3 dpf. $\boldsymbol{A}$, RT-PCR for zebrafish tnf $\alpha$ at $18,24,48,72,96$, and $120 \mathrm{hpf}$. thf $\alpha$ is clearly expressed at $18 \mathrm{hpf}$ and from 72 to $120 \mathrm{hpf}$, but expression may be 列 fish spinal cord. $\boldsymbol{E}$, Left, Cumulative histograms of glycinergic mIPSC amplitudes from control $(n=5)$ and TNF $\alpha$-treated $(n=5)$ embryos. Inset, Average mIPSC amplitude for the same conditions. Right, Average mIPSC waveforms for the same conditions.

Because CNQX and TNF $\alpha$ exhibit their effects at different stages in our protocols, we measured neural, muscular, and behavioral activity in $3 \mathrm{dpf}$ larvae treated with $\mathrm{TNF} \alpha$ or in $4 \mathrm{dpf}$ larvae treated with CNQX, the different stages at which glutamatergic mEPSCs scaled down and scaled up, respectively.

We examined the rhythmic input to MNs to investigate whether components of "fictive" swimming (in partially paralyzed embryos) (Drapeau et al., 1999) were disrupted at the spinal network level following perturbation of network activity. We found that the frequency of slow, swim episode-related oscillations in spinal neurons evoked by the application of $200 \mu \mathrm{M}$ NMDA (McDearmid and Drapeau, 2006) appeared to not be significantly different following chronic TNF $\alpha$ (control, $n=4$; TNF $\alpha, n=3 ; p=0.19$ ) or CNQX (control, $n=3$; CNQX, $n=3 ; p=0.71$ ) treatment (Fig. 6). Thus, slow oscillation frequencies at $3 \mathrm{dpf}$ were within the range of $0.7-1.1$ $\mathrm{Hz}$ for control and TNF $\alpha$-treated larvae, and in the range of $0.7-0.9$ $\mathrm{Hz}$ at $4 \mathrm{dpf}$ for CNQX-treated and control larvae.

Following this, we investigated the neuromuscular output of the spinal MNs onto muscle fibers in partially paralyzed embryos 
A

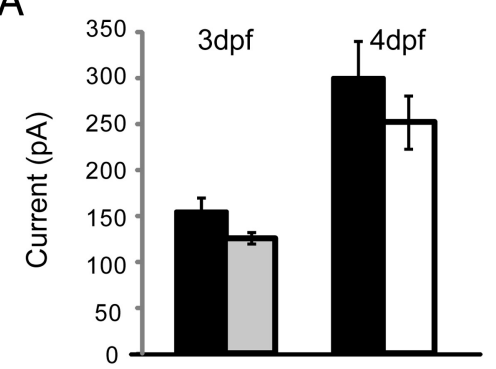

C
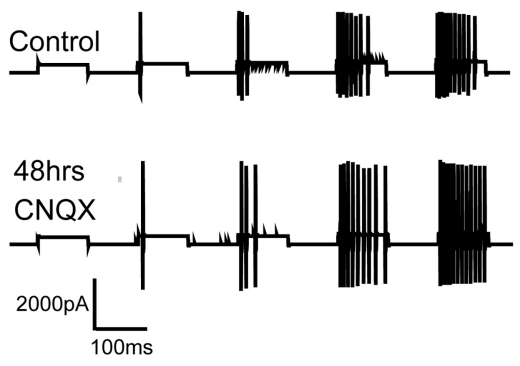

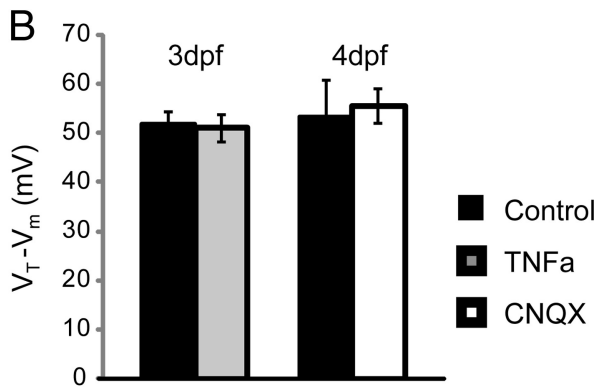

$\mathrm{D}$

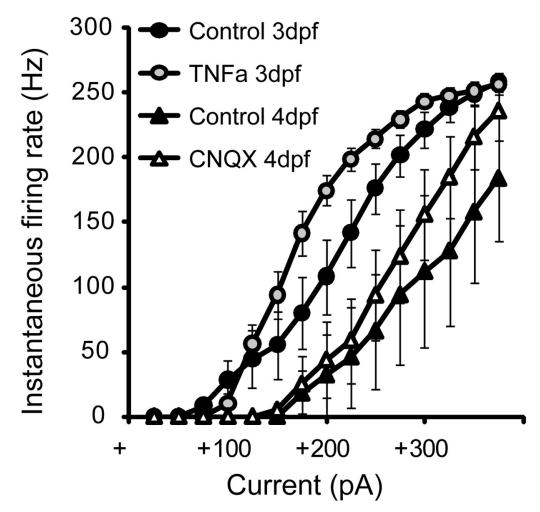

Figure 5. Chronic CNQX or TNF $\alpha$ treatment does not alter cellular excitability. $\boldsymbol{A}$, Rheobase current was not significantly different at $3 \mathrm{dpf}$ between control $(n=11)$ and TNF $\alpha$-treated $(n=10)$ larvae, at $4 \mathrm{dpf}$ between control $(n=6)$ and CNQX-treated $(n=8)$ larvae. $\boldsymbol{B}$, The average spike threshold $\left(V_{T}-V_{m}\right)$ was also unchanged after chronic TNF $\alpha$ or CNQX treatment under same conditions as $\boldsymbol{B}$. C, Representative spike trains evoked by somatic current injection steps ( $\Delta$ step $=25 \mathrm{pA}, 100$ ms duration, 15 steps total) in control and treated motoneurons at $4 \mathrm{dpf}$. $\boldsymbol{D}$, Average $f-I$ curves were also unchanged after chronic $\operatorname{TNF} \alpha$ or CNQX treatment under same conditions as $\boldsymbol{B}$.
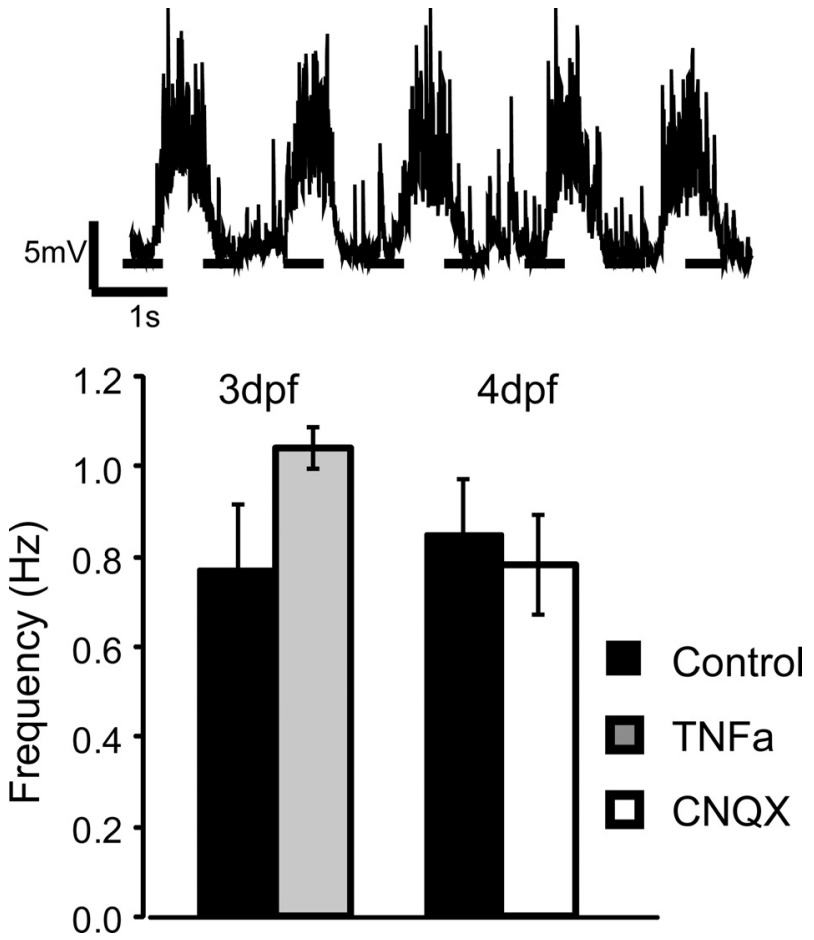

Figure 6. Slow oscillation frequency underlying fictive swimming in motoneurons is unchanged following chronic CNQX or TNF $\alpha$ treatment. Top, An example whole-cell currentclamp trace showing the slow oscillations elicited in a motoneuron by the extracellular application of $200 \mu \mathrm{m}$ NMDA. Bottom, Average frequency of NMDA-induced slow oscillations in motoneurons are not significantly different between control $(n=4)$ and TNF $\alpha$-treated $(n=3)$ larvae at $3 \mathrm{dpf}$, and control $(n=3)$ and CNQX-treated $(n=3)$ larvae at $4 \mathrm{dpf}$. (see Materials and Methods). The recordings were performed under the same conditions where synaptic scaling of glutamatergic mPSCs was seen in MNs. At $3 \mathrm{dpf}$, recordings of synaptic activity representing fictive bursts of swimming in slow-twitch (red) muscle were quantitatively compared between TNF $\alpha$-treated and control larvae (Fig. $7 A, B$ ). These bursts consist of a steady synaptic depolarization on which is superimposed a regular oscillation in synaptic intensity, with peaks that correspond to ipsilateral muscle contractions (Buss and Drapeau, 2001). The maximum synaptic amplitude of bursts was significantly reduced from control values by $\sim 40 \%$ in TNF $\alpha$-treated larvae (amplitudes of control and TNF $\alpha$ were $5.1 \pm 1.0$ and $8.5 \pm 1.0 \mathrm{mV}$, respectively; $p<0.05$ ), but no significant changes were seen from control values in burst duration or synaptic peak frequency (control, $n=11$; TNF $\alpha, n=9 ; p=0.90$ and 0.17 , respectively). At $4 \mathrm{dpf}$, recordings of synaptic activity representing episodes of fictive beat and glide swimming from slow-twitch muscle in CNQXtreated and control larvae were not significantly different from control in any parameter, including episode duration, beat duration, beat frequency, synaptic peaks per beat, synaptic peak frequency, maximum synaptic amplitude, or percentage time active (Fig. $7 C, D$ ) (control, $n=6$; CNQX, $n=8 ; p>0.05$ for all parameters).

Finally, under the same conditions where synaptic scaling of glutamatergic mPSCs was seen in MNs, we examined plasticity in the intact organism by looking at behavioral changes following activity perturbations in parameters of burst swimming at $3 \mathrm{dpf}$ and beat-and-glide swimming at $4 \mathrm{dpf}$ using high-speed video recordings (Fig. 8A). Quantitative analysis of burst swimming behavior of TNF $\alpha$-treated larvae at $3 \mathrm{dpf}$ revealed that their motor activity was not measurably different from controls in terms of tail contraction frequency (control, $n=3$; TNF $\alpha, n=5$; $p=$ 0.33 ) or free-swimming velocity (Fig. $8 B$ ) (control, $n=11$; $\mathrm{TNF} \alpha, n=13 ; p=0.95$ ). Contraction frequency was within the range of $36-40 \mathrm{~Hz}$ and average velocity was $\sim 28 \mathrm{~mm} / \mathrm{s}$ for both conditions at $3 \mathrm{dpf}$. Likewise, analysis of beat and glide swimming at $4 \mathrm{dpf}$ in CNQX-treated larvae revealed that their swimming behavior was not measurably different from controls in terms of tail contraction frequency (control, $n=8$; CNQX, $n=9 ; p=$ 0.51 ) or beat duration (Fig. $8 C$ ) (control, $n=8$; CNQX, $n=9$; $p=0.11$ ). Contraction frequency was close to $20 \mathrm{~Hz}$ at $4 \mathrm{dpf}$ while beat duration was $\sim 300-400 \mathrm{~ms}$ for both conditions at $4 \mathrm{dpf}$.

Together, these results indicate that normal levels of neural, muscular, and behavioral activity were maintained under conditions of upward or downward synaptic scaling.

Comparable acute changes in synaptic amplitudes do not disrupt network activity patterns

While the results presented above indicate that synaptic scaling may help stabilize motor activity patterns, it is possible if not likely that an intrinsic developmental program is equally important for motor development. To examine this latter possibility, 
we attempted to induce an increase or decrease in synaptic strength by acute pharmacological treatments and determine its impact on patterned motor network function. The treatments consisted of either immediately reducing synaptic strength with a low concentration of CNQX, or increasing synaptic amplitude by reducing AMPA receptor desensitization with CTZ (Trussell et al., 1993).

Preliminary electrophysiological experiments measuring the effect of acute CNQX treatment on glutamatergic mPSC amplitudes $(n=9)$ indicated that a concentration of $1 \mu \mathrm{M}$ CNQX reduced glutamatergic mPSC amplitudes by $\sim 15 \%$ $(n=5$; average amplitude at baseline and following CNQX was $20.7 \pm 1.2$ and $17.6 \pm 0.7 \mathrm{pA}$, respectively; $p<0.05$ ), as seen during scaling down following chronic TNF $\alpha$ treatment. Another set of preliminary experiments investigating the effects of acute CTZ treatment on glutamatergic mPSC amplitudes showed that $10 \mu \mathrm{M}$ CTZ acutely and significantly increased amplitudes by $\sim 25 \%(n=5$; average amplitude at baseline and following CTZ was $24.8 \pm 2.3$ and $30.2 \pm 1.9 \mathrm{pA}$, respectively; $p<0.05$ ).

To rapidly introduce these agents, we recorded patterned motor input to muscle cells in exposed preparations during acute perfusion with either $1 \mu \mathrm{M}$ CNQX or $10 \mu \mathrm{M}$ CTZ. At $3 \mathrm{dpf}$, recordings from slow-twitch muscle showed that acute application of CNQX was sufficient to significantly reduce maximum synaptic amplitude during episodes of fictive swimming by $\sim 25 \%$ ( $n=3$; amplitudes at baseline and following CNQX were $10.3 \pm 1.7$ and $7.5 \pm 1.1 \mathrm{pA}$, respectively; $p<0.05)$ but failed to affect burst length and synaptic peak frequency (Fig. $9 A, B$ ) ( $p=0.33$ and 0.42 , respectively). At $4 \mathrm{dpf}$, recordings from slow-twitch muscle showed that acute application of $10 \mu \mathrm{M}$ CTZ significantly increased the maximum synaptic amplitude during fictive swimming by $\sim 50 \%$ (Fig. $9 C, D)(n=10$; amplitudes at baseline and following CTZ were $2.3 \pm 0.4$ and $3.5 \pm 0.6 \mathrm{pA}$, respectively; $p<$ 0.01 ) and slightly but significantly increased the synaptic peak frequency as well (Fig. 9D) (average frequency at baseline and following CTZ was $22.2 \pm 0.6$ and $23.4 \pm 0.6 \mathrm{pA}$, respectively; $p<0.01)$. However, episode duration, beat duration, beat frequency, number of synaptic peaks per beat, and percentage time active remained unchanged (Fig. 9D) ( $p>0.05$ for all parameters). Together, these results indicate that the proper patterning of motor output can be maintained under conditions of altered synaptic amplitudes equivalent to those achieved by synaptic scaling.

\section{Discussion}

Previous studies have shown that neurons can respond homeostatically to reductions in network activity in a variety of ways,
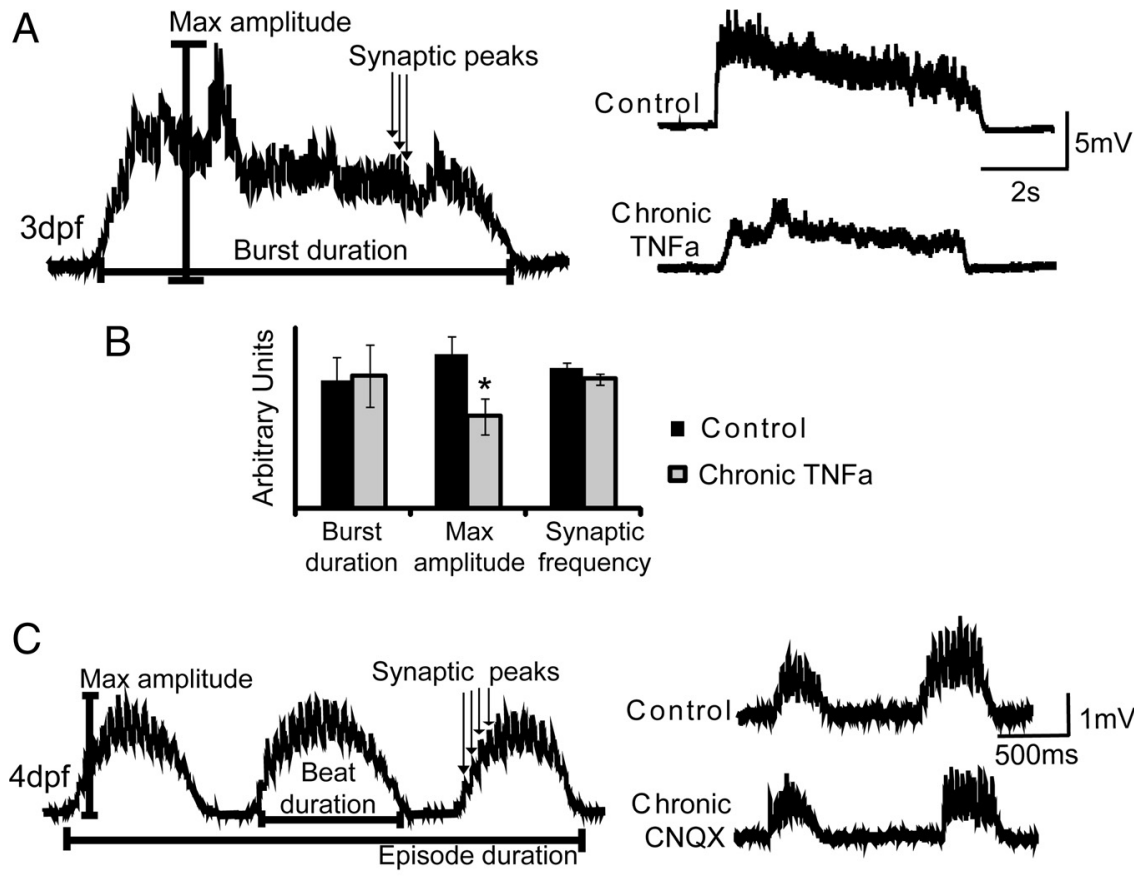

Chronic TNF $\alpha$ or CNQX treatment does not significantly alter the patterning of motor input to muscle cells at $3 \mathrm{dpf}$ or $n=11)$ and $n$ F $a$-treated ( $n=9)$ larvae* * $p<0.05$, Left, Example uscle fiber of fictive beat-and-glide swimming at $4 \mathrm{dpf}$. Analysis parameters are indicated. Right, Sample beat-and-glide recordsynaptic peak frequency, maximum synaptic amplitude, and percentage time active for slow-twitch muscle fibers at $4 \mathrm{dpf}$ for control $(n=6)$ and CNQX-treated $(n=8)$ larvae. None of the differences between conditions were significant $(p>0.05)$.

one of which is synaptic scaling. We have shown here that glutamatergic synaptic scaling occurs in the developing zebrafish and the behavioral implications are described for the first time in any preparation. In agreement with the literature, chronically reducing activity with either CNQX or TTX in vivo resulted in a significant and multiplicative scaling up (by $\sim 30 \%$ ) of glutamatergic mPSCs. However, in contrast to the scaling up observed following acute treatment with TNF $\alpha$ (Stellwagen et al., 2005; Stellwagen and Malenka, 2006), we observed that chronic TNF $\alpha$ treatment caused a significant and multiplicative scaling down (by 20\%) of glutamatergic mPSCs. Peripheral blockade of neuromuscular junctions was insufficient to induce scaling, indicating a requirement for alteration of central network activity. No effect was seen with AP-5 treatment or of TTX treatment on the amplitudes of glycinergic mPSCs, on the frequency of either type of mPSCs, or on intrinsic excitability of spinal neurons, although CNQX treatment caused an un-scaled reduction in mPSC amplitudes. Proper patterning of motor activity, as recorded in neurons, muscle cells, 
A

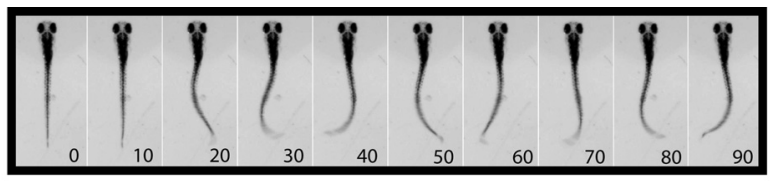

B 45 Frequency Velocity 3dpf

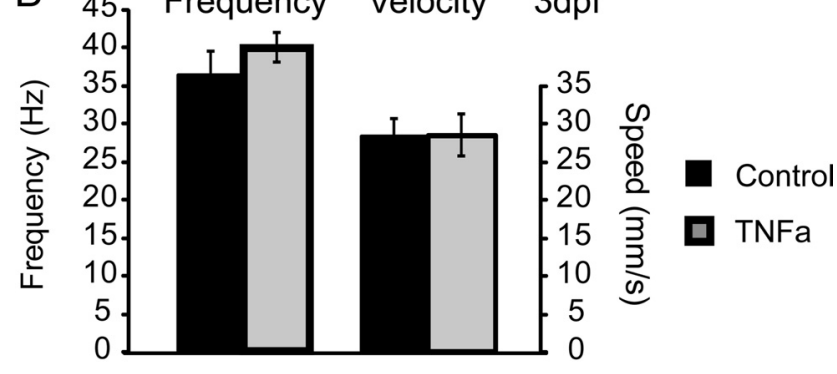

C

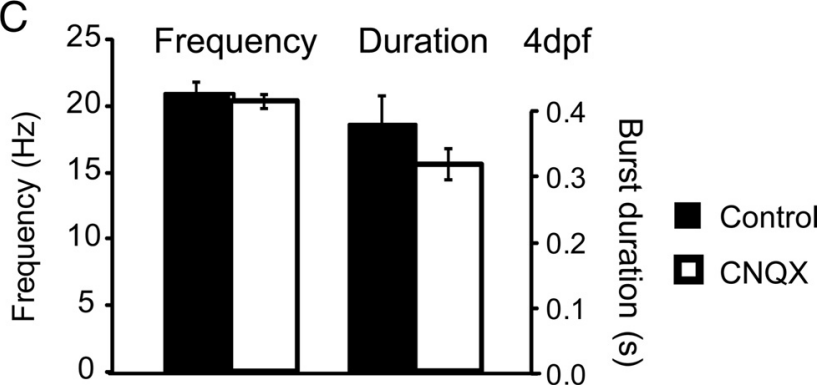

Figure 8. Larval swimming behavior is not altered following chronic TNF $\alpha$ or CNQX treatment. A, Swimming activity at 3-4 dpf is composed of high-frequency $(20-30 \mathrm{~Hz})$, lowamplitude tail contractions. In this example, one full cycle of swimming is observed from 50 to $80 \mathrm{~ms}$. The larva is immobilized in agarose, dorsal side up and anterior to the top of the image. Time of each frame is shown in the bottom right corner. $\boldsymbol{B}$, Left, Average swimming frequency of control ( $n=3)$ and TNF $\alpha$-treated $(n=5)$ larvae at $3 \mathrm{dpf}$. Right, Average free-swimming velocity under same conditions (control $n=11, \operatorname{TNF} \alpha$-treated $n=13$ ). C, Left, Average swimming frequency of control $(n=8)$ and CNQX-treated $(n=9)$ larvae at $4 \mathrm{dpf}$. Right, Average duration of swimming bursts under same conditions (control $n=8$, TNF $\alpha$-treated $n=9)$.

and in behavioral analyses, was maintained upon chronic synaptic scaling, either up or down, though the total synaptic amplitude may have changed. During comparable acute pharmacological alterations in synaptic strength, motor activity showed reduced total synaptic amplitude, but normal patterning persisted.

\section{Neuronal activity}

Our results and those published previously for other preparations (O’Brien et al., 1998; Turrigiano et al., 1998; Thiagarajan et al., 2005; Gonzalez-Islas and Wenner, 2006; Sutton et al., 2006; Echegoyen et al., 2007) showed an increase in synaptic strength at glutamatergic synapses following TTX or CNQX treatment. As TTX blocked all activity whereas CNQX blocked only transiently, this indicates that a reduction of activity is sufficient to induce the maximal scaling up observed upon complete block of activity. We observed a striking difference in the response of glutamatergic synapses to chronic TNF $\alpha$ treatment in our experiments. In cultured hippocampal neurons, acute exposure to $\mathrm{TNF} \alpha$ rapidly scales synapses up by $\sim 125 \%$ within 15 min (Stellwagen et al., 2005; Stellwagen and Malenka, 2006), while in our experiments they were significantly and multiplicatively scaled down after $24 \mathrm{~h}$ to $\sim 80 \%$ of control values. Interestingly, chronic TNF $\alpha$ treatment did result in a small but significant decrease in glutamatergic mPSC frequency of $\sim 20 \%$ from control values, but this may

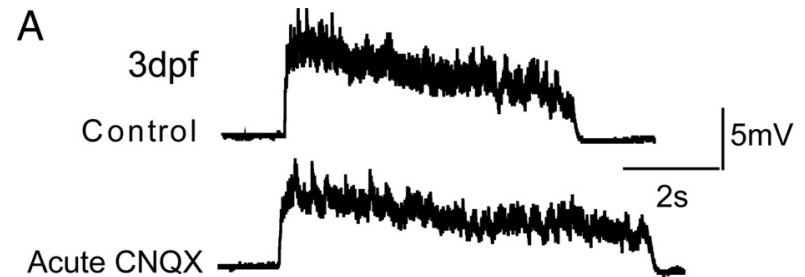

B
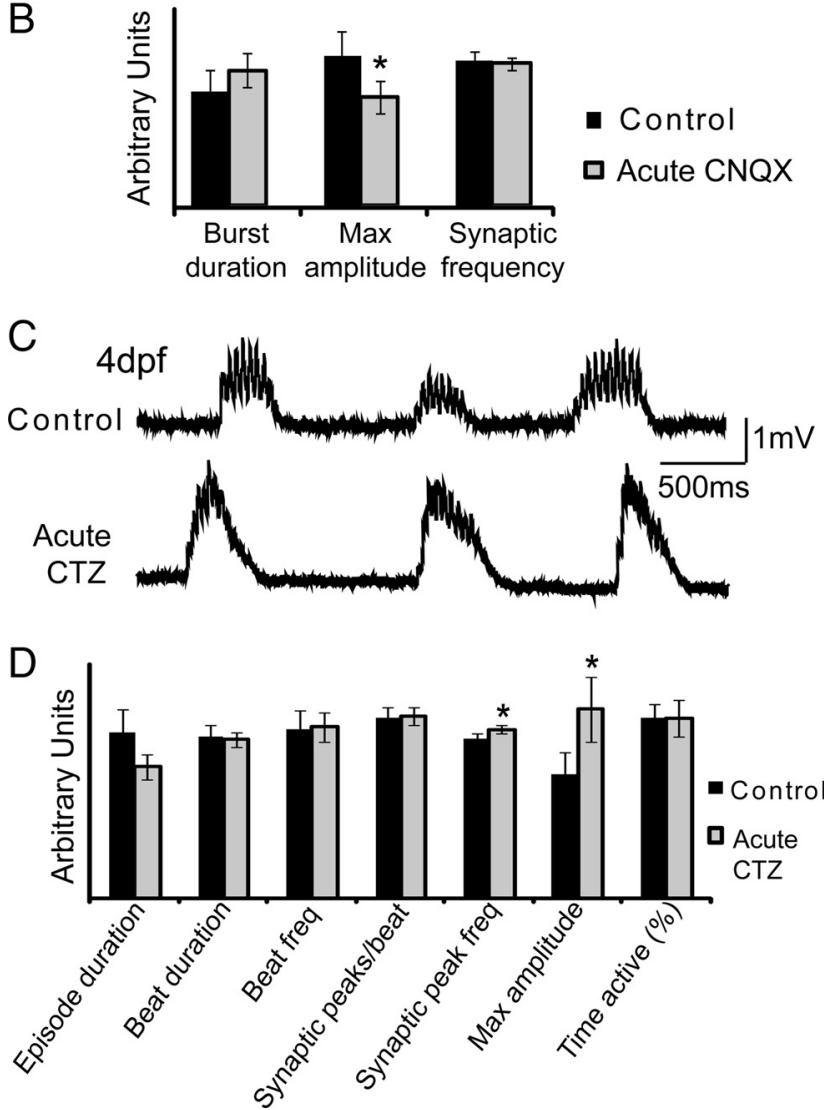

Figure 9. Acute CNQX or CTZ treatment significantly altered maximum synaptic amplitude (and contraction frequency of motor input - only in the second case) to muscle cells at $3 \mathrm{dpf}$ or $4 \mathrm{dpf}$, respectively. $A$, Sample fictive swimming burst recordings at $3 \mathrm{dpf}$ from a muscle fiber at baseline (control) and again 5 min after start of CNQX application. B, Average burst duration, maximum synaptic amplitude, and synaptic peak frequency at $3 \mathrm{dpf}$ for slow-twitch muscle fibers at baseline and following CNQX application $(n=3)$. Paired Student's $t$ test, ${ }^{*} p<0.05$. C, Sample fictive beat-and-glide swimming recordings at $3 \mathrm{dpf}$ from a muscle fiber at baseline (control) and again $5 \mathrm{~min}$ after start of CTZ application. $D$, Episode duration, burst duration, beat frequency, synaptic peaks per beat, synaptic peak frequency, maximum synaptic amplitude, and percentage time active at $4 \mathrm{dpf}$ for slow-twitch muscle fibers at baseline and following CNQX application $(n=10)$. Paired Student's $t$ test, ${ }^{* *} p<0.01$.

simply reflect an inability to detect some smaller amplitude events as they become obscured by background noise. Perhaps the differences in homeostatic responses to TNF $\alpha$ are due to differences in dose or time dependence in different preparations. Although the research to date has not shown that the same molecule is capable of mediating synaptic scaling bidirectionally, it is nonetheless possible that the concentration or timing of TNF $\alpha$ action could change the direction of synaptic scaling.

Although no significant scaling up or down of glycinergic mPSCs was seen following any of our treatments, other studies have provided evidence of activity-dependent synaptic scaling of glycinergic synapses in a homeostatic manner. Mongeon et al. (2008) demonstrated in vivo that evoked glycinergic IPSC ampli- 
tudes from spinal motor neurons in zebrafish shocked glial glycine transporter mutants scale down as the fish recover their ability to swim. Also, Whiting et al. (2009) showed that glycine receptor subunit expression in the rat cochlear nucleus was downregulated in vivo following monaural deprivation. Further investigations will be required to better elucidate the role of glycinergic synapses in mechanisms of homeostatic plasticity.

In addition to changes in synaptic properties, we also looked for possible changes in intrinsic cellular excitability following perturbations in network activity. However, the minimum current threshold required to produce an action potential, the spike voltage threshold, and the input-output relationship for current-induced firing were not significantly different in CNQX or TNF $\alpha$-treated larvae. The CNQX treatment results are in agreement with a recent study by Wilhelm et al. (2009) that showed no changes in cellular excitability in spinal neurons regardless of the duration of glutamatergic receptor blockade. In contrast, they found a significant increase in cellular excitability $12 \mathrm{~h}$ into chronic GABA receptor blockade as measured by rheobase current and spike threshold, and these changes decreased with time so that by $48 \mathrm{~h}$ after injection excitability was comparable to controls.

\section{Behavioral consequences}

Network activity is hypothesized to be maintained in the face of activity perturbation if synaptic scaling is a homeostatic process. Recordings of mature swimming behavior (at $3 \mathrm{dpf}$ following TNF $\alpha$ treatment and $4 \mathrm{dpf}$ following CNQX treatment) showed that no parameters of patterned swimming behavior were different from control at these stages. Similarly, our recordings from muscle fibers in TNF $\alpha$ - or CNQX-treated preparations showed no change from control in any temporal parameter of fictive swimming such as frequency or duration of swimming bursts, and no changes were measured in the slow oscillation component of rhythmic $\mathrm{MN}$ activity that are related to fictive swimming, consistent with a homeostatic maintenance of swimming activity. Acute pharmacological treatments that induced similar changes in synaptic strength and in the synaptic amplitude of motor output had no effect on the patterning of motor output. These latter results indicate that while synaptic scaling can be induced in the zebrafish spinal cord, synaptic patterning is more robust than synaptic strength during development and patterning can occur in the absence of homeostasis.

Certain caveats apply to the interpretation of our results in zebrafish spinal cord. We may have underestimated the extent of synaptic scaling up as we observed partial reversal of inhibition by CNQX. Nonetheless, Wilhelm and Wenner (2008) used a similar technique of a bolus injection of activity-reducing drugs in the in ovo chick spinal cord and measured the recovery of spontaneous network activity by mechanisms that include synaptic scaling. They showed that injection of a single receptor antagonist such as CNQX resulted in a temporary reduction in spontaneous network activity (as measured by immature limb movements) that recovered to control levels within hours. Nonetheless, we were surprised at the robustness of the patterned motor activity in muscle since near complete blockade by CNQX was required to suppress it, reflecting the resilience of the motor circuitry. However, in the absence of a behavioral measure of the consequences of acute changes in synaptic strength, we cannot exclude the possibility that these changes have significant behavioral consequences. Alternatively, it could be that global changes in excitatory transmission reset the entire system up or down and therefore leave the original balance intact and that they would have seen an impact had they induced a targeted change in excitability.

Activity-dependent scaling of glutamatergic synapses appears to be a ubiquitous neural phenomenon and helped compensate for changes in synaptic strength in the zebrafish motor network. However, synaptic scaling did not appear to be required to maintain the patterning of motor activity in vivo, which likely forms as a consequence of an intrinsic developmental program. It is interesting to note that complete blockade of glycinergic activity in zebrafish embryos has a drastic effect on neurogenesis, resulting in the loss of half the spinal neurons and indicating that there is a minimal activity dependence for these early steps of differentiation in development (McDearmid et al., 2006; Reynolds et al., 2008). The milder disruptions in glycinergic function observed in a glycine transporter mutant indicate that glycinergic strength is also susceptible to a form of synaptic homeostasis over a period of several days (Mongeon et al., 2008). Perhaps synaptic scaling may be required for maintaining a strong motor output and for motor development once the circuits become more dependent on feedback from the environment and descending control mechanisms.

\section{References}

Buss RR, Drapeau P (2000) Physiological properties of zebrafish embryonic red and white muscle fibers during early development. J Neurophysiol 84:1545-1557.

Buss RR, Drapeau P (2001) Synaptic drive to motoneurons during fictive swimming in the developing zebrafish. J Neurophysiol 86:197-210.

Buss RR, Bourque CW, Drapeau P (2003) Membrane properties related to the firing behavior of zebrafish motoneurons. J Neurophysiol 89:657-664.

Desai NS, Cudmore RH, Nelson SB, Turrigiano GG (2002) Critical periods for experience-dependent synaptic scaling in visual cortex. Nat Neurosci 5:783-789.

Drapeau P, Ali DW, Buss RR, Saint-Amant L (1999) In vivo recording from identifiable neurons of the locomotor network in the developing zebrafish. J Neurosci Methods 88:1-13.

Drapeau P, Saint-Amant L, Buss RR, Chong M, McDearmid JR, Brustein E (2002) Development of the locomotor network in zebrafish. Prog Neurobiol 68:85-111.

Echegoyen J, Neu A, Graber KD, Soltesz I (2007) Homeostatic plasticity studied using in vivo hippocampal activity-blockade: synaptic scaling, intrinsic plasticity and age-dependence. PLoS ONE 2:e700.

Gaiarsa J-L, Caillard O, Ben-Ari Y (2002) Long-term plasticity at GABAergic and glycinergic synapses: mechanisms and functional significance. Trends Neurosci 25:564-570.

Gonzalez-Islas C, Wenner P (2006) Spontaneous network activity in the embryonic spinal cord regulates AMPAergic and GABAergic synaptic strength. Neuron 49:563-575.

Horn D, Levy N, Ruppin E (1996) Neuronal-based synaptic compensation: A computational study in Alzheimer's disease. Neural Comput 8:1227-1243.

Kaneko M, Stellwagen D, Malenka RC, Stryker MP (2008) Tumor necrosis factor-[alpha] mediates one component of competitive, experiencedependent plasticity in developing visual cortex. Neuron 58:673-680.

Malenka RC, Bear MF (2004) LTP and LTD: an embarrassment of riches. Neuron 44:5-21.

McDearmid JR, Drapeau P (2006) Rhythmic motor activity evoked by NMDA in the spinal zebrafish larva. J Neurophysiol 95:401-417.

McDearmid JR, Liao M, Drapeau P (2006) Glycine receptors regulate interneuron differentiation during spinal network development. Proc Natl Acad Sci U S A 103:9679-9684.

Miller KD (1996) Synaptic economics: competition and cooperation in synaptic plasticity. Neuron 17:371-374.

Mongeon R, Gleason MR, Masino MA, Fetcho JR, Mandel G, Brehm P, Dallman JE (2008) Synaptic homeostasis in a zebrafish glial glycine transporter mutant. J Neurophysiol 100:1716-1723.

O’Brien RJ, Kamboj S, Ehlers MD, Rosen KR, Fischbach GD, Huganir RL (1998) Activity-dependent modulation of synaptic AMPA receptor accumulation. Neuron 21:1067-1078. 
Pawlak V, Schupp BJ, Single FN, Seeburg PH, Köhr G (2005) Impaired synaptic scaling in mouse hippocampal neurones expressing NMDA receptors with reduced calcium permeability. J Physiol 562:771-783.

Rabinowitch I, Segev I (2008) Two opposing plasticity mechanisms pulling a single synapse. Trends Neurosci 31:377-383.

Reynolds A, Brustein E, Liao M, Mercado A, Babilonia E, Mount DB, Drapeau P (2008) Neurogenic role of the depolarizing chloride gradient revealed by global overexpression of kcc2 from the onset of development. J Neurosci 28:1588-1597.

Roberts A, Li W-C, Soffe SR (2008) Roles for inhibition: studies on networks controlling swimming in young frog tadpoles. J Comp Physiol A Neuroethol Sens Neural Behav Physiol 194:185-193.

Saint-Amant L, Drapeau P (1998) Time course of the development of motor behaviors in the zebrafish embryo. J Neurobiol 37:622-632.

Saint-Amant L, Drapeau P (2001) Synchronization of an embryonic network of identified spinal interneurons solely by electrical coupling. Neuron 31:1035-1046.

Savioz A, Leuba G, Vallet PG, Walzer C (2009) Contribution of neural networks to Alzheimer disease's progression. Brain Res Bull 80:309-314.

Stellwagen D, Malenka RC (2006) Synaptic scaling mediated by glial TNF[alpha]. Nature 440:1054-1059.

Stellwagen D, Beattie EC, Seo JY, Malenka RC (2005) Differential regulation of AMPA receptor and GABA receptor trafficking by tumor necrosis factor-\{alpha\}. J Neurosci 25:3219-3228.
Sutton MA, Ito HT, Cressy P, Kempf C, Woo JC, Schuman EM (2006) Miniature neurotransmission stabilizes synaptic function via tonic suppression of local dendritic protein synthesis. Cell 125:785-799.

Thiagarajan TC, Lindskog M, Tsien RW (2005) Adaptation to synaptic inactivity in hippocampal neurons. Neuron 47:725-737.

Trussell LO, Zhang S, Raman IM (1993) Desensitization of AMPA receptors upon multiquantal neurotransmitter release. Neuron 10:1185-1196.

Turrigiano GG (2008) The self-tuning neuron: synaptic scaling of excitatory synapses. Cell 135:422-435.

Turrigiano GG, Leslie KR, Desai NS, Rutherford LC, Nelson SB (1998) Activity-dependent scaling of quantal amplitude in neocortical neurons. Nature 391:892-896.

Westerfield M (1995) The zebrafish book: a guide for laboratory use of zebrafish (Brachydanio rerio). Eugene, OR: University of Oregon.

Whiting B, Moiseff A, Rubio ME (2009) Cochlear nucleus neurons redistribute synaptic AMPA and glycine receptors in response to monaural conductive hearing loss. Neuroscience 163:1264-1276.

Wilhelm JC, Wenner P (2008) GABAA transmission is a critical step in the process of triggering homeostatic increases in quantal amplitude. Proc Natl Acad Sci U S A 105:11412-11417.

Wilhelm JC, Rich MM, Wenner P (2009) Compensatory changes in cellular excitability, not synaptic scaling, contribute to homeostatic recovery of embryonic network activity. Proc Natl Acad Sci U S A 106:6760-6765. 